\title{
Toward formal development of ML programs: foundations and methodology
}

\author{
- Extended abstract* -
}

\author{
Donald Sannella \\ Laboratory for Foundations of Computer Science \\ Department of Computer Science \\ University of Edinburgh
}

\author{
Andrzej Tarlecki \\ Institute of Computer Science \\ Polish Academy of Sciences \\ Warsaw
}

\begin{abstract}
A methodology is presented for the formal development of modular Standard ML programs from specifications. Program development proceeds via a sequence of design (modular decomposition), coding and refinement steps. For each of these three kinds of step, conditions are given which ensure the correctness of the result. These conditions seem to be as weak as possible under the constraint of being expressible as "local" interface matching requirements.
\end{abstract}

\section{Introduction}

The ultimate goal of work on algebraic specification is to provide a formal basis for program development to support a methodology for the systematic evolution of programs from specifications by means of verified refinement steps. In this paper we present such a methodology aimed at the development of programs in the Standard ML programming language [HMM 86], [Har 86]. We are interested particularly in the semantic and foundational underpinnings of modular program development and in formulating precise conditions which ensure the correctness of refinement steps, rather than in informal rules governing good programming practice. We build on previous work as follows:

Algebraic specifications: The most fundamental assumption in work on algebraic specification is the view of software systems as algebras, abstracting away from details of algorithms and code and focussing on data representation and functional behaviour of programs. A specification describes some class of algebras, defining in this indirect way which programs are acceptable as realisations. Whatever specification formalism we use, we assume that any specification $S P$ determines an algebraic signature $S i g[S P]$ and a class of algebras Mod[SP] over this signature, called the models of SP. See [ST 85a].

Behavioural equivalence: It may be argued that a software system should be accepted as a realisation of a specification $S P$ as long as it "behaves like" a model of $S P$ even if it does not satisfy $S P$ exactly. This intuition may be made precise by introducing an appropriate notion of behavioural equivalence of algebras. Then the interpretation of $S P$ may be relaxed modulo this behavioural equivalence. See [GGM 76], [Rei 81], [GM 82], [ST 87] and [NO 88].

Specification refinement: A theory of formal program development by stepwise refinement of specifications requires a precise definition of the notion of refinement and when a refinement step is considered to be correct. In the following paragraph we summarize the work on this topic presented in

*This is a very much abridged version of [ST 89]. This version ornits proofs, many technicalities, all examples and much of the motivation and background. 
[ST 88b]; other relevant papers include [GB 80], [Ehr 82], [EKMP 82], [Wand 82], [Gan 83], [Lip 83], [Ore 83] and many others.

The simplest notion of refinement of one specification $S P 1$ to another $S P 2$ would only require inclusion of model classes, i.e. $\operatorname{Mod}[S P 2] \subseteq \operatorname{Mod}[S P 1]$. A more realistic view involves a construction $\kappa$ taking models of $S P 2$ to models of $S P 1$; we write $S P 1 \leadsto S P 2$. Here, a construction is just a function $\kappa: A l g(\operatorname{Sig}[S P 2]) \rightarrow A l g(\operatorname{Sig}[S P 1])$ between classes of algebras. Using these so-called constructor implementations, the program development process consists of a sequence of consecutive implementation steps, $\left.S P_{0} \underset{\kappa_{1}}{\longrightarrow} S P_{1} \widetilde{\kappa}_{2}>\cdots{\widetilde{\kappa_{n}}}_{n}\right\rangle S P_{n}$, where $S P_{0}$ is the original high-level specification of requirements. Then, the composition of constructions $\kappa_{n} ; \cdots ; \kappa_{2} ; \kappa_{1}$ forms a "parameterised program" (cf. [Gog 84]) which implements $S P_{0}$ in terms of $S P_{n}$.

Extended ML: The specification language Extended ML [ST 85b,86] is a vehicle for formal development of programs in the programming language Standard ML. Extended ML enhances Standard ML by allowing more information in module interfaces (axioms in ML signatures) and less information in module bodies (axioms in place of code in ML structures/functors). Standard ML forms a subset of Extended ML, since Standard ML datatype and function definitions are just axioms of a certain special form. Thus Extended ML is a wide-spectrum language in the spirit of CIP-L [Bau 85].

Data abstraction in modular programming: A general theory of modular program development using data abstraction is presented in [Sch 86]. The main issue, referred to as "the correctness problem of data abstraction", is why it is possible for the implementor of a specification to provide a realisation which is correct only up to behavioural equivalence, while users of the result may view it as if it satisfied the specification exactly. A very rough explanation of this apparent paradox is that users are not able to take advantage of the properties which distinguish "exact" models of a specification from their behavioural approximations. It is argued that this property, called stability, should be required of any programming language designed to support data abstraction.

The central observation which led to the ideas presented here was that Standard ML functors may be used to code constructions in the above sense. Also, Extended ML allows us to specify such constructions before they are coded. ML's modularisation facilities guarantee the composability of such constructions by analogy with function composition. This gives a view of program development which is more complex but methodologically more appealing than the one in [ST 88b].

A programming task is presented as an Extended ML functor heading, i.e. an Extended ML signature $S P_{n}$ specifying structures to which the functor may be applied, and an Extended ML signature $S P_{0}$ specifying the required result structure. Recall that Extended ML signatures may contain axioms. Rather than proceeding from $S P_{0}$ to $S P_{1}, \ldots$, and then from $S P_{n-1}$ to $S P_{n}$ as described above, we take a more global view with development steps of the following kinds:

Design step: Sketch the implementation process $S P_{0} \underset{\kappa_{1}}{\longrightarrow} S P_{1} \underset{\kappa_{2}}{\longrightarrow} \cdots \widetilde{\kappa}_{n} S S P_{n}$ without coding the constructions $\kappa_{1}, \ldots, \kappa_{n}$. This gives rise to specifications of functors $\kappa_{1}, \ldots, \kappa_{n}$ which are then viewed as separate programming tasks in their own right to which the same methodology applies. The composition of these functors results in a construction which implements $S P_{0}$ in terms of $S P_{n}$. The design may have a more complex structure than this linear notation suggests, since functors may have multiple arguments and the same functor may be used in different places.

Coding step: Code a construction by providing a functor body in the form of an encapsulated structure containing type and value definitions. It is also possible to use an "abstract program" here, i.e. an Extended ML functor body containing axioms. 
Refinement step: Further refine abstract programs in a stepwise fashion by providing successively more concrete (but possibly still non-executable) versions which fill in some of the decisions left open by the more abstract version.

The paper is organized as follows. Section 2 gives an overview of the modularisation facilities of Standard ML and reviews the main features of the Extended ML specification language. Section 3 recalls the notion of behavioural equivalence and introduces the new notion of behavioural consequence which plays a basic role in verification conditions ensuring the correctness of development steps. Some preliminary results are given for proving behavioural consequence between loose specifications; as far as we know this topic has not been directly addressed in the literature. Section 4 sketches the semantics of Extended ML functors. The concept of universal correctness of an Extended ML functor with respect to its interface specifications is introduced following [Sch 86]. A functor is universally correct if it produces a result which satisfies the output interface up to behavioural equivalence whenever it is given an argument satisfying the input interface up to behavioural equivalence.

Sections 5 and 6 present the methodology of program development. Section 5 discusses design steps in which a functor is defined by decomposition into a collection of simpler functors. One representative case is studied and verification conditions ensuring the correctness of the decomposition are given. Section 6 is about coding and refinement steps. Following [Sch 86], we present universal correctness as the conjunction of three properties: simple correctness, simple consistency and stability. A functor is simply correct if it produces a result which satisfies the output interface up to behavioural equivalence whenever it is given an argument which exactly satisfies the input interface. Stability is assumed to be ensured for Standard ML functors since Standard ML is designed to support data abstraction. We give verification conditions which guarantee simple correctness of directly coded functors and functors produced by successive refinement steps. Thus, once a final Standard ML functor is obtained it will be simply correct and stable (and simply consistent since it is a program), and hence universally correct.

\section{An overview of Extended ML}

The version of Extended ML used in this paper differs in certain details from the one presented in [ST 85b,86]. The changes were motivated by the methodological issues to be discussed in this paper.

The reader need not be acquainted with the features and syntactic details of Standard ML itself. It will be sufficient to know that a sequence of Standard ML declarations defines a set of types and values, where some values are functions and others are constants. A complete description of the language appears in [Mil 86], and a formal semantics is in [HMT 87].

Extended ML is based on the modularisation facilities for Standard ML [MacQ 86]. These facilities are designed to allow large Standard ML programs to be structured into modules with explicitlyspecified interfaces. In Standard ML, interfaces (called signatures) and their implementations (called structures) are defined separately. Every structure has a signature which gives the names of the types and values defined in the structure. Structures may be built on top of existing structures, so each one is actually a hierarchy of structures. Certain built-in types and values are pervasive - they may be regarded as forming a structure Perv which is automatically included as a substructure of every signature and structure. Functors ${ }^{1}$ are "parameterised" structures; the application of a functor to a structure yields a structure. A functor has an input signature describing structures to which it may be applied, and an output signature describing the result of an application. It is sometimes necessary, in

\footnotetext{
${ }^{I}$ Category theorists should note that ML functors have no morphism part, and that ML supports no explicit notion of morphism between structures.
} 
order to allow interaction between different parts of a program, to declare that certain substructures (or just certain types and/or values ${ }^{2}$ ) in the hierarchy are identical or shared.

To make signatures more useful as interfaces of structures in program specification and development, we allow them to include axioms which put constraints on the permitted behaviour of the components of the structure. In order to allow different stages in the evolution of a program to be expressed in the same framework, we allow structures and functor bodies to contain a mixture of ML code and non-executable axioms.

In Standard ML and the version of Extended ML described in [ST 85b,86], a functor interface is taken to be the signatures in the heading augmented by inferred sharing (sharing by construction in [MacQ 86]). For methodological reasons, we view the interface of a functor as containing only the information explicitly given in the functor heading. (Thus functors in Extended ML are actually parameterised abstractions in the sense of [MacQ 86].) To make this regime work in practice, we allow functor headings to contain sharing constraints declaring that certain components of the result are inherited from the parameters. We will assume that Extended ML signatures used as functor result specifications do not include references to external structures. This is purely for simplicity of presentation; our methodology (including all correctness results) can be extended to handle this case.

Signatures and structures both denote classes of algebras. To be more exact, each signature or structure determines a many-sorted algebraic signature where sorts are type names and operation names are value names and the typing of values determines the rank of operation names. Because of type definitions like type $t=s$ in structures and sharing constraints in signatures, in general there may be several names for a single type or value. We cope with this by assuming that the names which occur in the algebraic signature associated with a structure or signature are unique internal semantic-level names which are associated with one or more external identifiers which may appear in Extended ML text. Two types or values share iff they have the same internal identifier. A structure or signature determines the class of algebras over its associated (internal) algebraic signature which satisfy its axioms; recall that code in structures is just a sequence of axioms of a certain special form.

The role of signatures as interfaces suggests that they should not distinguish between behaviourally equivalent algebras. Nevertheless, axioms in signatures are treated "literally" as in structures. Only when a signature is used as an interface, is behavioural abstraction used to relax its interpretation by closing under behavioural equivalence with respect to an appropriate set of types, which thus may depend on the context in which the signature is used. This extra flexibility turns out to be crucial for the methodology we develop in this paper.

Extended ML is actually entirely independent of Standard ML (although not of Standard ML's modularisation facilities): the semantics of Extended ML in [ST 86] was parameterised by an arbitrary institution [GB 84] which means that we are free to adopt any logical system for writing specifications. This not only allows us to use any desired specification style but also to adopt any programming language with an algebraic-style formal definition for writing code. We are not going to follow this line: we present our ideas in the framework of total many-sorted algebras, using a purely functional subset of Standard ML without polymorphism or higher-order functions for writing code. We can see no obvious obstacles to prevent generalisation to the institutional framework.

\section{Behavioural equivalence}

Definition 3.1 Let $\Sigma$ be an algebraic signature with a distinguished set $O B S \subseteq$ sorts $(\Sigma)$ of observable sorts. Suppose $A, B$ are $\Sigma$-algebras with $|A|_{s}=|B|_{s}$ for all $s \in O B S$. $A$ and $B$ are behaviourally

\footnotetext{
${ }^{2}$ Standard ML does not support sharing declarations for values. Extended ML supports this on the grounds that it is easier and more uniform to treat types and values in the same way.
} 
equivalent with respect to $O B S$, written $A \equiv O B S B$, if for any term $t$ of a sort in $O B S$ containing only variables $X$ of sorts in $O B S$ and any valuation $v: X \rightarrow|A|_{O B S}\left(=|B|_{O B S}\right), t_{A}(v)=t_{B}(v)$.

There is a model-theoretic formulation of this definition due to [Sch 86] (Thm. 4.4.6) which is useful for proving that two specific algebras are behaviourally equivalent. However, in formal program development we are rarely faced with this problem. Rather, we want to know that two loose specifications match up to behavioural equivalence.

Definition 3.2 Let $\Sigma$ be an algebraic signature with a distinguished set of observable sorts $O B S \subseteq$ sorts $(\Sigma)$. Let $S P 1$ and $S P 2$ be specifications over $\Sigma$, let $A$ be a $\Sigma$-algebra, and let $K$ be a class of $\Sigma$-algebras. $A$ satisfies $S P 2$ up to behavioural equivalence with respect to $O B S$, written $A=O B S S P 2$, if there exists an algebra $B \in \operatorname{Mod}[S P 2]$ such that $A \equiv O B S B$. $K$ satisfies $S P 2$ up to behavioural equivalence with respect to $O B S$, written $K={ }^{O B S} S P 2$, if every algebra in $K$ satisfies $S P 2$ up to behavioural equivalence w.r.t. $O B S . S P 2$ is a behavioural consequence of $S P 1$ with respect to $O B S$, written $S P 1=^{O B S} S P 2$, if $\operatorname{Mod}[S P 1] \vDash{ }^{O B S} S P 2$.

A typical situation which involves proving behavioural consequence is checking whether an Extended ML structure fits an Extended ML signature. We do not require that the structure satisfies the axioms in the signature literally, but only up to behavioural equivalence with respect to an appropriate set of observable sorts. For top-level structures the sorts corresponding to pervasive types are taken as observable. For structures occurring inside functor bodies, it is appropriate to take additionally some sorts in the functor parameters as observable. In both cases, we require the signature to be a behavioural consequence of the structure, except that we permit the algebraic signature associated with the structure to be "larger" than the one associated with the signature.

As far as we know, the important problem of proving that one specification is a behavioural consequence of another has not been addressed directly in the literature although some related material may be found in [Gan 83], [Rei 84], [Sch 86], [ST 87] and [NO 88]. The following results address this problem by giving proof-theoretic sufficient conditions for behavioural equivalence. More work needs to be done here but the theorems below cover the most obvious cases including those which are normally considered in work on algebraic specification.

Consider two specifications $S P 1$ and $S P 2$ over the same algebraic signature $\Sigma$ (i.e. $S i g[S P 1]=$ $\operatorname{Sig}[S P 2]=\Sigma)$ and a set $O B S \subseteq \operatorname{sorts}(\Sigma)$ of observable sorts.

Proposition 3.3 If $S P 1 \models S P 2$ then $S P 1 \models{ }^{O B S} S P 2$.

The following results depend on the notion of observable consequences [ST 87] of a conditional equation $\varphi$, defined syntactically as conditional equations with the same premises as $\varphi$ and with the conclusion of $\varphi$ translated by a context to an observable sort (details in [ST 89]). We write ObsCon $(\Phi)$ for the set of all observable consequences of conditional equations in $\Phi$.

Theorem 3.4 Suppose SP2 is given as a set $\Phi$ of conditional $\Sigma$-equations with premises of observable sorts. Then $S P 1 \models^{O B S} S P 2$ iff $S P 1 \models O b s C o n(\Phi)$.

Corollary 3.5 Suppose $S P 2$ is given as a set $\Phi$ of $\Sigma$-equations. Then $S P 1 \vDash{ }^{O B S} S P 2$ iff $S P 1 \vDash$ $O b s \operatorname{Con}(\Phi)$.

Corollary 3.6 Suppose SP2 is given as a set $\Phi$ of conditional $\Sigma$-equations with premises of observable sorts. Let $\Phi=\Phi 1 \cup \Phi 2$. Then, if $S P 1 \models \Phi 1$ and $S P 1 \models O b s C o n(\Phi 2)$ then $S P 1 \models{ }^{O B S} S P 2$. 
If $S P 2$ has non-trivial structure, the problem is harder; see [Far 89].

As we mentioned earlier, checking that an Extended ML structure STR fits an Extended ML signature $S I G$ involves proving behavioural consequence between two specifications over different algebraic signatures. According to the Standard ML matching rules, STR may contain more components then $S I G$, hence the algebraic signature $\Sigma_{S T R}$ associated with $S T R$ may be larger than the algebraic signature $\Sigma_{S I G}$ associated with $S I G$. Moreover, because $S T R$ is permitted to share more than $S I G$ requires, the real requirement is that a quotient of $\Sigma_{S I G}$ is a sub-signature of $\Sigma_{S T R}$. It is important to decide which of the two algebraic signatures will provide the operations we can use to build observable terms.

Definition 3.7 Given specifications $S P 1$ and $S P 2$, an algebraic signature morphism $\sigma: S i g[S P 2] \rightarrow$ $\operatorname{Sig}[S P 1]$, and a set of sorts $O B S \subseteq \operatorname{sorts}(\operatorname{Sig}[S P 2])$, we say that $S P 2$ is a behavioural consequence of $S P 1$ with respect to $O B S$ via $\sigma$, written $S P 1 \models_{\sigma}^{O B S} S P 2$, if derive from $S P 1$ by $\sigma \models^{O B S} S P 2$ where derive is a specification-building operation such that $S i g$ [derive from $S P 1$ by $\sigma]=S i g[S P 2]$ and $\operatorname{Mod}[$ derive from $S P 1$ by $\sigma]=\{A 1|\sigma| A 1 \in \operatorname{Mod}[S P 1]\}[$ ST 88a].

Notation In the rest of this paper we write $S P 1 \models_{S i g[S P 2]}^{O B S} S P 2$ or even $S P 1 \vDash{ }^{O B S} S P 2$ since $\sigma$ will be unambiguously determined by the context. We use a similar convention for individual algebras.

An alternative would be to consider a set of observable sorts $O B S^{\prime} \subseteq \operatorname{sorts}(\operatorname{Sig}[S P 1])$, and define $S P 1 \models_{\sigma}^{O B S^{\prime}} S P 2$ to mean $S P 1 \models^{O B S^{\prime}}$ translate $S P 2$ by $\sigma$ where translate is a specification-building operation such that $\operatorname{Sig}[$ translate $S P 2$ by $\sigma]=S i g[S P 1]$ and $M o d[$ translate $S P 2$ by $\sigma]=\{A 1 \in$ $\left.\operatorname{Alg}(\operatorname{Sig}[S P 1])|A 1|_{\sigma} \in \operatorname{Mod}[S P 2]\right\}$ [ST 88a]. We have chosen the more permissive possibility:

Fact 3.8 If $S P 1 \models^{\sigma(O B S)}$ translate $S P 2$ by $\sigma$ then derive from $S P 1$ by $\sigma \models^{O B S} S P 2$.

\section{Semantics of functors}

Consider a Standard ML functor

functor $F\left(X: S I G_{\text {in }}\right): S I G_{\text {out }}$ sharing sharing-decl $=B O D Y$.

The Standard ML signatures $S I G_{\text {in }}$ and $S I G_{\text {out }}$ determine algebraic signatures $\Sigma_{\text {in }}$ and $\Sigma_{\text {out }}$. These are not disjoint in general. Their common part $\Sigma_{s h r}=\Sigma_{i n} \cap \Sigma_{o u t}$ with inclusions $\iota_{i n}: \Sigma_{s h r} \hookrightarrow \Sigma_{i n}$ and $\iota_{\text {out }}: \Sigma_{s k r} \hookrightarrow \Sigma_{\text {out }}$ expresses the sharing requirements in the functor heading.

Since $B O D Y$ is just Standard ML code, it determines the basic semantics of the functor $F$ as a function $F_{\text {bsem }}: \operatorname{Alg}\left(\Sigma_{\text {in }}\right) \rightarrow \operatorname{Alg}\left(\Sigma_{\text {out }}\right)$ which for any algebra $A \in A \lg \left(\Sigma_{\text {in }}\right)$, builds an algebra $F_{b s e m}(A) \in A l g\left(\Sigma_{\text {out }}\right)$ such that $\left.F_{b s c m}(A)\right|_{\Sigma_{s h r}}=\left.A\right|_{\Sigma_{s h r}}$.

The complete picture is a bit more complex. The argument for $F$ may be a much larger structure $S T R_{\text {arg }}$ with algebraic signature $\Sigma_{\text {arg }}$, which may in addition contain more sharing than required by the functor input signature. The matching rules of the language will determine an algebraic signature morphism $\sigma: \Sigma_{i n} \rightarrow \Sigma_{\text {arg }}$. Any identification $\sigma$ makes on $\Sigma_{i n}$ must be preserved when the functor $F$ is applied to $S T R_{\text {arg }}$. The following technicalities capture this idea.

For any algebraic signature morphism $\sigma: \Sigma_{\text {in }} \rightarrow \Sigma_{\text {arg }}$, the translation of $\Sigma_{\text {arg }}$ by $F$ via $\sigma$, written $F\left(\Sigma_{\text {arg }}[\sigma]\right)$, and the translation of $\sigma$ by $F$, written $F[\sigma]$, are defined by the following pushout:

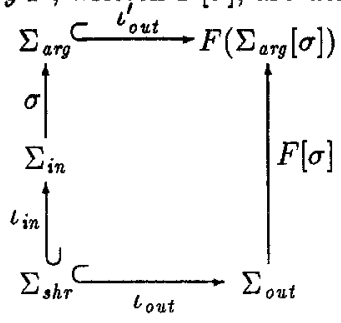


Any $\Sigma_{\text {arg }}$-algebra $A$ may be "fitted" as an argument for $F$ using $\sigma:\left.A\right|_{\sigma}$ is a $\Sigma_{\text {in }}$-algebra to which we can apply $F_{b s e m}$. The requirement on $F_{b s e m}$ ensures that $\left.F_{b s e m}\left(\left.A\right|_{\sigma}\right)\right|_{\Sigma_{s h r}}=\left.\left(\left.A\right|_{\sigma}\right)\right|_{\Sigma_{s h r}}$. Thus, there is a unique $F\left(\Sigma_{\text {arg }}[\sigma]\right)$-algebra $F_{\text {gres }}(A[\sigma])$ such that $\left.F_{\text {gres }}(A[\sigma])\right|_{\Sigma_{\text {arg }}}=A$, and $\left.F_{\text {gres }}(A[\sigma])\right|_{F[\sigma]}=$ $F_{\text {bsem }}\left(\left.A\right|_{\sigma}\right)$. We refer to $F_{\text {gres }}(A[\sigma])$ as the global result of the application of $F$ to $A$.

The global result of functor application is "larger" than indicated in Section 2. We expect a structure over the output signature as a result. However, the sharing between those components of the actual parameter that occur in the output must be preserved. Thus, the result of applying $F$ to $A$, written $F_{\text {res }}(A[\sigma])$, is the reduct $\left.F_{\text {gres }}(A[\sigma])\right|_{F[\sigma]\left(\Sigma_{\text {out }}\right)}$ of the global result to the signature $F[\sigma]\left(\Sigma_{\text {out }}\right)$.

Above, we have adopted a "local" view of the algebraic signature $\Sigma_{\text {arg }}$ and algebra $A$, in which they model the structure to which the functor is actually applied. There is also a "global" view, suggested by the fact that we develop a modular Standard ML program by defining a collection of interrelated structures. The resulting structure environment may be viewed as a single structure having all the top-level structures as substructures. We may interpret $A$ and $\Sigma_{\text {arg }}$ in the above as representing this whole structure. It seems to be necessary to adopt this view since sharing may take place between separate structures in the environment, and thus some structure which is not included in the actual parameter may nonetheless provide some additional means of manipulating values of the shared types.

The above semantics of Standard ML functors may be carried over to Extended ML functors as well, but we have to cope with a few additional issues. Consider an Extended ML functor

$$
\text { functor } F\left(X: S I G_{\text {in }}\right): S I G_{\text {out }} \text { sharing sharing-decl }=B O D Y \text {. }
$$

Recall that $S I G_{\text {in }}, S I G_{\text {out }}$ and $B O D Y$ may contain axioms, and so are in fact specifications with $\Sigma_{\text {in }}=$ $\operatorname{Sig}\left[S I G_{\text {in }}\right], \Sigma_{\text {out }}=\operatorname{Sig}\left[S I G_{\text {out }}\right]$ and $\Sigma_{\text {body }}=\operatorname{Sig}[B O D Y]$, and $\operatorname{Mod}\left[S I G_{\text {in }}\right] \subseteq \operatorname{Alg}\left(\Sigma_{\text {in }}\right), \operatorname{Mod}\left[S I G_{\text {out }}\right] \subseteq$ $\operatorname{Alg}\left(\Sigma_{\text {out }}\right)$ and $\operatorname{Mod}[B O D Y] \subseteq \operatorname{Alg}\left(\Sigma_{\text {body }}\right)$. The comments above concerning the relationship between $\Sigma_{\text {in }}$ and $\Sigma_{\text {out }}$ still apply. Moreover, we have an algebraic signature morphism $\tau: \Sigma_{\text {out }} \rightarrow \Sigma_{\text {body }}$ (this allows the body to contain more components than required by the output signature and for extra sharing between the components the output will contain) and an inclusion $\iota_{b}: \Sigma_{\text {shr }} \hookrightarrow \Sigma_{\text {body }}$ such that $\iota_{b}=\iota_{\text {out }} ; \tau$. As with Standard ML functors, we require that the shared part of the input is preserved by the body. This was a trivial requirement for Standard ML functors since Standard ML code does not allow the programmer to modify the input. In Extended ML, however, this may lead to inconsistency since the body may impose new requirements on the input.

The basic semantics $\mathcal{F}_{\text {bsem }}: A \lg \left(\Sigma_{i n}\right) \rightarrow \wp\left(A l g\left(\Sigma_{\text {out }}\right)\right)$ of the above Extended ML functor assigns to any $\Sigma_{i n}$-algebra a class of $\Sigma_{o u t}$-algebras determined by $B O D Y$ such that for any $A \in \operatorname{Alg}\left(\Sigma_{i n}\right)$ :

$$
\mathcal{F}_{b s e m}(A)=\left\{\left.B\right|_{\tau} \mid B \in \operatorname{Mod}[B O D Y] \text { and }\left.B\right|_{\Sigma_{\text {in }} \cap \Sigma_{\text {body }}}=\left.A\right|_{\Sigma_{\text {in }} \cap \Sigma_{\text {body }}}\right\} \text {. }
$$

The domain of $F$ is defined as follows: $\operatorname{Dom}(F)=\left\{A \in A \lg \left(\Sigma_{i n}\right) \mid \mathcal{F}_{b \text { sem }}(A) \neq \emptyset\right\}$.

We will identify the function $\mathcal{F}_{\text {bsem }}$ with the family of partial functions $F_{\text {bsem }}: A l g\left(\Sigma_{\text {in }}\right) \simeq A \lg \left(\Sigma_{\text {out }}\right)$ such that $F_{\text {beem }}(A)$ is defined exactly when $A \in \operatorname{Dom}(F)$ and then $F_{b s e m}(A) \in \mathcal{F}_{\text {bsem }}(A)$. Hence, $F_{\text {bsem }}(A)$ will stand for an arbitrary algebra in $\mathcal{F}_{\text {bsem }}(A)$. We will refer to both $\mathcal{F}_{\text {bsem }}$ and any $F_{\text {bsem }}$ as basic semantic functions, where the context and the font will determine which notion is being used.

The above definition of the basic semantics of Extended ML functors properly generalises the basic semantics of Standard ML functors. The only difference is that in Extended ML the code need not be executable, and it need not define the result unambiguously (it may even be inconsistent, in which case no result exists). Just as before, we can extend each of the basic semantic functions $F_{b s e m} \in \mathcal{F}_{b s e m}$ to the partial semantic functions $F_{\text {gres }}$ and $F_{\text {res }}$ operating on any algebra matching the input signature.

Notation For any Extended ML functor we use all the above notation without recalling it explicitly. 
The basic semantics of Extended ML functors disregards the fact that signatures may contain axioms, taking account only of the axioms in the body. Rather than take axioms in signatures into consideration when defining the above "operational" semantics of functors, we introduce a notion of correctness meant to model the idea that functors should fulfill the requirements in their headings.

For a functor to be correct we will require that if the input structure satisfies the requirements imposed by the input signature then the functor produces result structure(s) which satisfy the requirements stated in the output signature. Axioms in signatures should be considered only up to behavioural equivalence w.r.t. a pre-specified set of primitive types. In Extended ML we take those to be exactly the built-in pervasive types sorts(Perv). We require that the structure produced by applying a functor to a given input structure satisfies the output requirements not necessarily literally but only up to behavioural equivalence. Thus we have to accept the possibility that the requirements in the input signature are not satisfied literally, but again only up to behavioural equivalence. It is not enough to consider the input and output signature separately from contexts in which the functor may potentially be used ${ }^{3}$. Looking just at the input or output signature as it stands yields very few non-trivial "observations". However, when the functor is used and the input types are instantiated in a richer context, the user usually has many more ways to observe the types of the resulting structure. Behavioural equivalence must be considered at a global level: at the level of the environment in which the actual input structure resides and to which the result structure is added. The following definition follows almost directly the notion of universal implementation of [Sch 86].

Definition 4.1 An Extended ML functor of the form

$$
\text { functor } F\left(X: S I G_{i n}\right): S I G_{\text {out }} \text { sharing sharing-decl }=B O D Y
$$

is universally correct if for any algebraic (argument) signature $\Sigma_{\text {arg }}$ and fitting morphism $\sigma: \Sigma_{\text {in }} \rightarrow$ $\Sigma_{\text {arg }}$, any $\Sigma_{\text {arg }}$-algebra $A$ such that $A=^{\text {sorts(Perv) }}$ translate $S I G_{i n}$ by $\sigma$ and any $F_{b s e m} \in \mathcal{F}_{b s e m}$ :

1. $\left.A\right|_{\sigma} \in \operatorname{Dom}(F)$;

2. $F_{\text {gres }}(A[\sigma]) \vDash^{\text {sorts(Perv) }}$ translate $S I G_{\text {out }}$ by $F[\sigma]$; and

3. for any $\Sigma_{a r g}$-algebra $B$ such that $B \equiv_{\text {sorts (Perv) }} A$ and $\left.B\right|_{\sigma} \models S I G_{\text {in }}$, there exists a $F\left(\Sigma_{a r g}[\sigma]\right)$ algebra $\widehat{B}$ such that $\left.\hat{B}\right|_{\iota_{\text {out }}^{\prime}}=B, \hat{B} \equiv_{\text {sorts }(\operatorname{Pexv})} F_{\text {gres }}(A[\sigma])$ and $\left.\widehat{B}\right|_{F[\sigma]} \models S I G_{\text {out }}$.

A careful reader may have realized that condition 3 entails condition 2 (and more implicitly, condition 1 as well). We have stated these conditions separately since conditions 1 and 2 are what one intuitively expects while condition 3 turns out to be required for technical reasons in situations in which a programming task is decomposed into separate but interacting subtasks.

In our methodology, a programming task is presented as an Extended ML functor heading. The programmer is to produce a functor body consisting of Standard ML code such that the functor is universally correct. In the sequel we present methods for achieving this goal by modular decomposition and stepwise refinement with explicit conditions which ensure the correctness of the result.

\section{System design: functor decomposition}

In this section we concentrate on defining functors as a composition of simpler functors, i.e. by modular decomposition. The idea is simple: just come up with a bunch of other functors, and define the

\footnotetext{
${ }^{3}$ In [ST 85b] we have proposed a semantics based on such a view of functors and signatures as "closed" entities. We now consider this to be a mistake and propose a different view, better suited as a basis for the methodology we develop.
} 
functor being implemented as an expression over these functors. These functors can then themselves be implemented using the same technique of modular decomposition or by supplying an "abstract program" (see Section 6). We need to impose appropriate verification conditions to ensure that:

- The expression is well-formed: functors in the expression are always applied to structures whose signatures match their input signatures, and the result signature matches the output signature.

- The functor definition is correct: roughly, for any argument satisfying the input signature, the result produced satisfies the output signature (modulo the discussion concerning behavioural equivalence in Section 4).

We will sketch just one simple but representative case of functor decomposition in which a functor is defined as the sequential composition of two simpler functors. In [ST 89] we give full details and analyze both a simpler case (unitary decomposition) and a more complex case (parallel decomposition). The general situation is discussed as well.

Consider an Extended ML functor

$$
\text { functor } F\left(X: S I G_{i n}\right): S I G_{\text {out }} \text { sharing sharing-decl }=G 2(G 1(X))
$$

where $G 1$ and $G 2$ are functors with headings

$$
\begin{aligned}
& \text { functor } G 1\left(Y 1: S I G 1_{\text {in }}\right): S I G 1_{\text {out }} \text { sharing sharing-decl1 } \\
& \text { functor } G 2\left(Y 2: S I G 2_{\text {in }}\right): S I G 2_{\text {out }} \text { sharing sharing-decl2. }
\end{aligned}
$$

Then the basic semantics of $F$ is (roughly) the composition of the basic semantics of $G 1$ and $G 2$.

To ensure that the definition of $F$ is correct, we have to verify that two conditions are satisfied:

- The definition of $F$ is well-formed according to the Standard ML typechecking rules:

- A quotient of $\Sigma 1_{i n}$ is a subsignature of $\Sigma_{i n}$ (given by a morphism $\tau_{i n}: \Sigma 1_{\text {in }} \rightarrow \Sigma_{\text {in }}$ ).

- A quotient of $\Sigma 2_{\text {in }}$ is a subsignature of $\Sigma 1_{\text {out }}$ (given by a morphism $\tau_{\text {inter }}: \Sigma 2_{\text {in }} \rightarrow \Sigma 1_{\text {out }}$ ).

- A quotient of $\Sigma_{\text {out }}$ is a subsignature of $\Sigma 2_{\text {out }}$ (given by a morphism $\tau_{\text {out }}: \Sigma_{\text {out }} \rightarrow \Sigma 2_{\text {out }}$ ).

- The sharing between $\Sigma_{\text {in }}$ and $\Sigma_{\text {out }}$ follows (by composition) from the sharing between $\Sigma 1_{\text {in }}$ and $\Sigma 1_{o u t}$ and between $\Sigma 2_{\text {in }}$ and $\Sigma 2_{o u t}$ (as indicated by morphisms $\rho 1: \Sigma_{o h r} \rightarrow \Sigma 1_{\text {shr }}$ and $\left.\rho 1: \Sigma_{s h r} \rightarrow \Sigma 2_{s h r}\right)$.

This gives rise to the following commutative diagram:

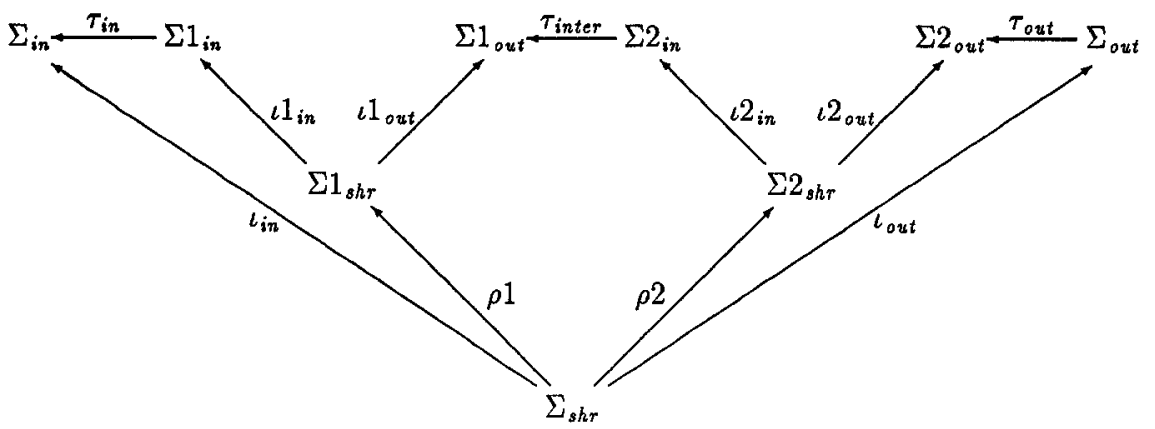

- The requirements stated in the functor interfaces match one another: 
- $S I G_{\text {in }}$ entails $S I G 1_{\text {in }}$ up to behavioural equivalence.

- $S I G 1_{\text {out }}$ entails $S I G 2_{\text {in }}$ up to behavioural equivalence.

- $S I G 2_{\text {out }}$ entails $S I G_{\text {out }}$ up to behavioural equivalence.

Theorem 5.1 Consider Extended $M L$ functors $F, G 1$ and $G 2$ as above. Suppose that the definition of $F$ is well-formed according to the Standard ML typechecking rules, determining a commutative diagram as above. Suppose that the following conditions are satisfied:

$$
\begin{aligned}
& \text { 1. } S I G_{\text {in }} \models_{\Sigma 1_{\text {in }}}^{\text {sorts }\left(\left(1_{\text {in }}\left(\rho 1\left(\Sigma_{\text {shr }}\right)\right)\right)\right.} S I G 1_{\text {in }} \\
& \text { 2. } S I G 1_{\text {out }} \models_{\left.\Sigma 2_{\text {in }}^{\text {sorts }\left(l 2_{\text {in }}\right.}\left(\rho 2\left(\Sigma_{\text {shr }}\right)\right)\right)}^{\text {s. }} S I G 2_{\text {in }} \\
& \text { 3. } S I G 2_{\text {out }} \models_{\left.\Sigma_{\text {out }}^{\text {sorts }\left(l_{\text {out }}\right.}\left(\Sigma_{\text {shr }}\right)\right)} S I G_{\text {out }}
\end{aligned}
$$

Then, if $G 1$ and $G 2$ are universally correct then so is $F$.

Proof idea Consider any algebraic signature $\Sigma_{a r g}$, fitting morphism $\sigma: \Sigma_{i n} \rightarrow \Sigma_{a r g}$ and $\Sigma_{a r g}$-algebra $A$. The global result of the application of $F$ to $A$ is defined via the basic semantics of $F$ :

$$
\mathcal{F}_{\text {bsem }}\left(\left.A\right|_{\sigma}\right)=\left\{\left.A 2\right|_{\tau_{\text {out }}} \mid A 2 \in \mathcal{G} 2_{\text {bsem }}\left(\left.A 1\right|_{\tau_{\text {inter }}}\right) \text { for some } A 1 \in \mathcal{G} 1_{\text {bsem }}\left(\left.\left(\left.A\right|_{\sigma}\right)\right|_{\tau_{\text {in }}}\right)\right\}
$$

Rather than using this directly to prove the universal correctness of $F$, one has to apply $G 1$ and $G 2$ consecutively at the global level and show that the global result of $F$ is a reduct of the global result obtained. Then universal correctness of $G 1$ and $G 2$ may be used to show that the consecutive global results satisfy appropriate translations of the corresponding interfaces up to behavioural equivalence. The details of the construction and the proof may be found in [ST 89].

We would not expect formal development to proceed in practice without backtracking, mistakes and iteration, and we do not claim to remove the possibility of unwise design decisions. For example, it may be necessary to adjust the interfaces in the above decomposition both in order to achieve the verification conditions 1-3 and to resolve problems which arise later while implementing $G 1$ and G2. If a decomposition has been proved correct then some changes to the interfaces may be made without affecting correctness: for example, $S I G 1_{\text {out }}$ may be strengthened or $S I G 2_{\text {in }}$ may be weakened without problems. It is also possible to weaken $S I G_{\text {out }}$ or strengthen $S I G_{i n}$. This will preserve the correctness of the decomposition but since it changes the specification of $F$ it must be cleared with $F$ 's clients (higher-level functors which use $F$ and/or the customer). Once we have made such a change we can also strengthen $S I G 1_{\text {in }}$ or weaken $S I G 2_{\text {out }}$ correspondingly in order to take advantage of the modification. Then overall correctness is still assured since the remaining interfaces are unaffected.

Functors correspond to (parameterised) abstract data types. We are free to change the implementation (body) of a functor at any time. As long as the new implementation is universally correct with respect to the functor heading, this change is invisible to the rest of the program. This is ensured since explicit interfaces insulate a functor implementation from its use.

\section{System design: refinement of abstract programs}

The previous section discussed conditions under which functors can be correctly implemented by decomposition into simpler functors. At some point it is necessary to actually write code to implement a functor. In this section we discuss how correct code can be developed gradually by means of stepwise refinement of loose abstract programs. Our goal is to arrive at a functor body containing only executable code which is universally correct with respect to the given functor heading. 


\subsection{Simple correctness and stability}

Although the notion of universal correctness expresses the correctness property one should aim at in program development, it is very inconvenient as a basis for verification of abstract programs as pointed out in [Sch 86]. First of all, we are not allowed to rely on the input specification literally, but only on its observable consequences. Second, we are required to consider all possible structures to which the functor may be applied rather than considering just structures over the input signature. A solution presented in [Sch 86] is to split universal correctness into three properties which will be ensured separately. Consider an Extended ML functor of the form

$$
\text { functor } F\left(X: S I G_{i n}\right): S I G_{o u t} \text { sharing sharing-decl }=B O D Y \text {. }
$$

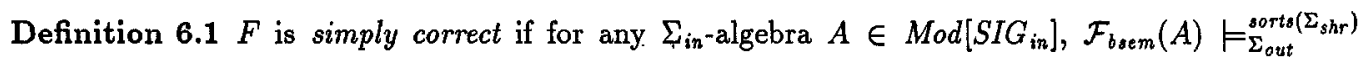
$S I G_{\text {out }}$.

Definition $6.2 F$ is simply consistent if $\operatorname{Mod}\left[S I G_{\text {in }}\right] \subseteq \operatorname{Dom}(F)$.

Definition 6.3 $F$ is stable if for any algebraic signature $\Sigma_{\text {arg }}$ and fitting morphism $\sigma: \Sigma_{\text {in }} \rightarrow \Sigma_{\text {arg }}$, any $\Sigma_{\text {arg }}$-algebras $A, B$ such that $A \equiv_{\text {sorts(Perv })} B$, and for any $A^{\prime} \in \mathcal{F}_{\text {gres }}(A[\sigma])$ there exists $B^{\prime} \in \mathcal{F}_{\text {gres }}(B[\sigma])$ such that $A^{\prime} \equiv{ }_{\text {sorts(Perv) }} B^{\prime}$.

The main idea behind the definition of stability is that a functor is stable if and only if it preserves behavioural equivalence.

Theorem 6.4 An Extended ML functor is universally correct whenever it is simply correct, simply consistent and stable.

Simple correctness is a property which can be verified "statically" in the sense that we do not have to consider all the different ways in which the functor can be applied. It is enough to consider only structures over the input signature. Moreover, while verifying simple correctness we are allowed to pretend that the input structure satisfies the input signature literally. This is therefore a condition which we will expect a user of our methodology to verify for each of the functors he defines.

Stability is a different matter. This property should be guaranteed by the designer of the programming language used. Any language which is designed to support data abstraction should ensure that only stable functors (modules, packages, clusters, etc.) are definable. See [Sch 86] for a much more complete discussion of this issue.

Working hypothesis Every functor definable in Standard ML is stable.

Discussion We could turn this working hypothesis into a theorem for the purely functional subset of Standard ML we are using here, under the type discipline sketched in Section 2. To prove the corresponding theorem, or even state it precisely, for full Standard ML would require developing an integrated algebraic view of (at least) exceptions, polymorphism, higher-order functions, imperative features, partial functions and non-terminating functions. This is an important long-term goal which we are confident may be achieved, but it is orthogonal to the issues discussed in this paper.

Under the above hypothesis, any simply correct functor whose body is coded in Standard ML is universally correct (recall that every Standard ML functor is defined for all structures over its algebraic input signature, and so is obviously simply consistent). However, this is not guaranteed for Extended ML functors in general, and it would not be reasonable to expect this of any specification language. Extended ML functors arising during the development process need not be universally 
correct; our methodology guarantees only that they are simply correct by requiring refinement steps to preserve this property. Consequently, when we arrive at a Standard ML functor it will be simply correct and simply consistent, and it will be stable by the above working hypothesis, and hence by Theorem 6.4 it will be universally correct.

One might argue that simple consistency is a requirement which should be imposed on every Extended ML functor which arises in the program development process. This would seem to prevent blind alleys in program development. But even a total functor may have no computable (or acceptably efficient) realisation, so we cannot hope to avoid blind alleys in general anyway. It is advisable to check for simple consistency at each stage of development but this is not required for correctness.

\subsection{Abstract programs}

The conclusion of the discussion in the previous subsection is that the user's only obligation is to produce code for the functor body in such a way that the resulting functor definition is simply correct. The user may begin by writing a loose abstract program and then gradually refine this in a stepwise manner until a version containing only Standard ML code is obtained.

The following theorem gives the condition which the first version (and in fact all versions) of the body must satisfy in order to ensure simple correctness of the functor.

Theorem 6.5 An Extended $M L$ functor of the form

$$
\text { functor } F\left(X: S I G_{i n}\right): S I G_{\text {out }} \text { sharing sharing-decl }=B O D Y
$$

is simply correct if and only if

$$
\text { (translate (derive from } \left.S I G_{\text {in }} \text { by } \iota_{s i} \text { ) by } \iota_{s b}\right) \cup B O D Y \models_{\Sigma_{\text {out }}}^{\text {sorts }\left(\iota_{\text {out }}\left(\Sigma_{s h r}\right)\right)} S I G_{\text {out }}
$$

where $\iota_{s i}: \Sigma_{i n} \cap \Sigma_{b o d y} \hookrightarrow \Sigma_{i n}$ and $\iota_{s b}: \Sigma_{i n} \cap \Sigma_{b o d y} \hookrightarrow \Sigma_{b o d y}$ are the algebraic signature inclusions. ${ }^{4}$

We could employ this theorem to check the simple correctness of each version of the functor body obtained as a result of successive refinement steps. But in practice this is inconvenient since subsequent versions of the body will become increasingly more detailed and lower level, making it difficult to relate them in a simple way to the output interface. It is much more natural to relate each new version of the functor body directly with the previous one. Then we can exploit the simple correctness of the previous version to establish the simple correctness of the new version as follows:

Corollary 6.6 If an Extended ML functor of the form

$$
\text { functor } F\left(X: S I G_{\text {in }}\right): S I G_{\text {out }} \text { sharing sharing-decl }=B O D Y
$$

is simply correct and

(translate (derive from $S I G_{i n}$ by $\left.\iota_{s i}^{\prime}\right)$ by $\left.\iota_{s b}^{\prime}\right) \cup B O D Y^{\prime} \models_{\Sigma_{b o d y}}^{s o r t s\left(\iota_{s b}\left(\Sigma_{i n} \cap \Sigma_{b o d y}\right) \text { ) }\right.} B O D Y$ where $\iota_{s i}^{\prime}: \Sigma_{i n} \cap \Sigma_{b o d y^{\prime}} \hookrightarrow \Sigma_{i n}$ and $\iota_{s b}^{\prime}: \Sigma_{i n} \cap \Sigma_{b o d y^{\prime}} \hookrightarrow \Sigma_{b o d y^{\prime}}$, then the functor is simply correct if $B O D Y$ is replaced by $B O D Y^{\prime}$ as well.

\footnotetext{
${ }^{4}$ The horrible expression on the left-hand side of the entailment should be thought of as $S I G_{\text {in }} \cup B O D Y$ (and similarly for Corollary 6.6).
} 


\subsection{Hierarchically structured abstract programs}

The previous subsection only treated the special case of "flat" abstract programs, i.e. abstract programs not containing substructures. Substructures provide a way to structure functor bodies into conceptual units, in additional to the means already provided by functor decomposition. Structuring functor bodies in this way also gives a corresponding structure to the verification process.

Hitherto we have strictly adhered to a regime of insulating system units from their clients by means of interfaces (Extended ML signatures). Now, the units of interest are substructures of functor bodies and their clients are the functor bodies themselves. Syntactically, this naturally leads to the requirement that Extended ML substructure declarations always explicitly include the Extended ML signature which the substructure is supposed to fit. Just as before, we view this signature as containing all the information available about the substructure.

This view of substructures means that we can view them as (calls of) locally-defined parameterless functors. The verification conditions are thus very much reminiscent of those we stated for functor decomposition. The only difference is that substructures implicitly import the part of the functor body which precedes the substructure declaration. There is no interface at this point insulating the substructure from the details of the preceding code. See [ST 89] for details.

\section{Conclusions and future work}

In this paper we have sketched a methodology for the formal development of programs supported by the modularisation facilities of Standard ML [MacQ 86], [HMT 87]. Our starting point was the specification language Extended ML [ST 85b,86]. The present work may be viewed as an adaptation to the Extended ML framework of some of the ideas in [Sch 86] amalgamated with our ideas on implementation of specifications [ST 88b] developed in the context of ASL [SWi 83], [ST 88a].

We have borrowed from [Sch 86] the technical concepts of universal correctness (Section 4) and simple correctness and stability (Section 6) and the thesis that it is proper to demand that stability be guaranteed by the programming language. We have generalised his results on composition of universally correct functors by allowing interfaces to match up to behavioural equivalence rather than requiring them to match literally (Section 5). Our verification conditions seem to be as weak as possible under the constraint of being expressible as "local" signature matching requirements. We have also given some preliminary results for proving that interfaces match up to behavioural equivalence (Section 3).

From [ST 88b] we take the concept of constructor implementation and the idea that constructors play a central role in program development. As hinted in the conclusion of [ST 88b], constructors correspond to Standard ML functors. In the Extended ML framework developed here we allow such constructors to be specified before they are actually coded. Implementing an Extended ML functor heading by functor decomposition amounts to sketching the entire constructor implementation process for that functor. Because the constructors involved are specified, the correctness of this decomposition may be verified before any code is written.

The soundness of our methodology depends on the stability of the target programming language. This must be checked for the subset of ML we use in this paper and for other potential target languages.

The aims of this work are broadly similar to those of work on rigorous program development by the VDM school (see e.g. [Jones 80]). VDM is a method which has been widely applied in practice. However, it lacks formal mathematical foundations and explicit structuring mechanisms. The RAISE project [BDMP 85] is attempting to fill these gaps. This can be seen as converging with our current work which builds on formal mathematical foundations with a strong emphasis on structure of spec- 
ifications, and attempts to address problems of practical usability. Two advantages of our approach are the use of behavioural equivalence which handles the transition between data specification and representation in a more general way than VDM's retrieve functions, and the use of institutions to obtain independence from the underlying logical framework and target programming language.

A notion of modular specification related to the one in Extended ML is developed in a series of papers beginning with [EW 85]. The underlying semantic notions seem to be close although there are many technical differences and the main issues of concern differ as well. While [EW 85] and later papers mainly discuss the module concept itself and operations for manipulating modules with compatibility results, in Extended ML these are taken as given since they are inherited from Standard ML. Recent work on system development in that framework [EFHLP 87] builds around notions of realization and refinement which seem to be based on different intuitions than the ones we try to model here.

The eventual practical feasibility of formal program development depends on the existence of an integrated support system. There is a need for (at least) a parser and typechecker for Extended ML specifications, a theorem prover, a refinement step verifier, some capability for rapid prototyping, and appropriate environmental tools. Most of the technology on which such a system depends has already been developed so that constructing it would mostly be a matter of applying and integrating existing techniques rather than inventing new ones.

Acknowledgements: The work presented here is based on the ideas developed by Oliver Schoett in [Sch 86]. Thanks to Jordi Farrés, Mike Fourman and the anonymous TAPSOFT' 89 referees for comments on a draft. This work has been partially supported by grants from the Polish Academy of Sciences and from the U.K. Science and Engineering Research Council. The bulk of the work was done during a visit by the second author to Edinburgh University under the support of an SERC Visiting Fellowship.

\section{References}

[Bau 85] Bauer, F.L. et al (the CIP language group). The Wide Spectrum Language CIP-L. LNCS 183.

[BDMP 85] Bjørner, D., Denvir, T., Meiling, E. and Pedersen, J.S. The RAISE project: fundamental issues and requirements. Report RAISE/DDC/EM/1/V6, Dansk Datamatic Center.

[Ehr 82] Ehrich, H.-D. On the theory of specification, implementation, and parametrization of abstract data types. Journal of the Assoc. for Computing Machinery 29, 206-227.

[EFHLP 87] Ehrig, H., Fey, W., Hansen, H., Löwe, M. and Parisi-Presicce, F. Algebraic theory of modular specification development. Technical report, Technische Univ. Berlin.

[EKMP 82] Ehrig, H., Kreowski, H.-J., Mahr, B. and Padawitz, P. Algebraic implementation of abstract data types. Thearetical Computer Science 20, 209-263.

[EW 85] Ehrig, H. and Weber, H. Algebraic specification of modules. In: Formal Models in Programming (E.J. Neuhold and G. Chronist, eds.). North-Holland, 231-258.

[Far 89] Farrés, J. Forthcoming Ph.D. thesis, Univ, of Edinburgh.

[Gan 83] Ganzinger, H. Parameterized specifications: parameter passing and implementation with respect to observability. Trans. Prog. Lang. Syst. 5, 318-354.

[GGM 76] Giarratana, V., Gimona, F. and Montanari, U. Observability concepts in abstract data type specification. Proc. 5th Intl. Symp. on Mathematical Foundations of Computer Science, Gdaǹsk. LNCS 45, 576-587.

[Gog 84] Goguen, J.A. Parameterized programming. IEEE Trans. Software Engineering SE-10, 528-543.

[GB 80] Goguen, J.A. and Burstall, R.M. CAT, a system for the structured elaboration of correct programs from structured specifications. Technical report CSL-118, Computer Science Laboratory, SRI International.

[GB 84] Goguen, J.A. and Burstall, R.M. Introducing institutions. Proc. Logics of Programming Workshop, CarnegieMellon, LNCS 164, 221-256. 
[GM 82] Goguen, J.A. and Meseguer, J. Universal realization, persistent interconnection and implementation of abstract modules. Proc. 9th Intl. Collog. on Automata, Languages and Programming, Aarhus. LNCS 140, $265-281$.

[Jones 80] Jones, C. Software Development: A Rigorous Approach. Prentice-Hall.

[Har 86] Harper, R. Introduction to Standard ML. Report ECS-LFCS-86-14, University of Edinburgh.

[HMM 86] Harper, R., MacQueen, D.B. and Milner, R. Standard ML. Report ECS-LFCS-86-2, Univ. of Edinburgh.

[HMT 87] Harper, R., Milner, R. and Tofte, M. The semantics of Standard ML (version 1). Report ECS-LFCS-87-36, Univ, of Edinburgh.

[Lip 83] Lipeck, U. Ein algebraischer Kalkül für einer strukturierten Entwurf von Datenabstraktionen. Ph.D. thesis, Abteilung Informatik, Universität Dortmund.

[MacQ 86] MacQueen, D.B. Modules for Standard ML. In [HMM 86].

[Mil 86] Milner, $R$. The Standard ML core language (revised). In [HMM 86].

[NO 88] Nivela, M.P. and Orejas, F. Initial behaviour semantics for algebraic specifications. Proc. 5th Workshop on Specification of Abstract Data Types, Gullane, Scotland, LNCS 332, 184-207.

[Ore 83] Orejas, F. Characterizing composability of abstract interpretations. Proc. Intl. Conf. on Foundations of Computation Theory, Borgholm, Sweden, LNCS 158, 335-346.

[Rei 81] Reichel, H. Behavioural equivalence - a unifying concept for initial and final specification methods. Proc. 3rd Hungarian Computer Science Conference, Budapest, 27-39.

[Rei 84] Reichel, H. Behavioural validity of equations in abstract data types. Contributions to General Algebra 3, Proc. of the Vienna Conference. Teubner, 301-324.

[ST 85a] Sannella, D. and Tarlecki, A. Some thoughts on algebraic specification. Proc. 3rd Workshop on Theory and Applications of Abstract Data Types, Bremen. Springer Informatik-Fachberichte Vol. 116, 31-38.

[ST 85b] Sannella, D. and Tarlecki, A. Program specification and development in Standard ML. Proc. 12th ACM Symp. on Principles of Programming Languages, New Orleans, 67-77.

[ST 86] Sannella, D. and Tarlecki, A. Extended ML: an institution-independent framework for formal program development. Proc. Workshop on Category Theory and Computer Programming, LNCS 240, 364-389.

[ST 87] Sannella, D. and Tarlecki, A. On observational equivalence and algebraic specification. J. Comp. and Sys. Sciences 34, 150-178.

[ST 88a] Sannella, D. and Tarlecki, A. Specifications in an arbitrary institution. Information and Computation 76, 165-210.

[ST 88b] Sannella, D.T. and Tarlecki, A. Toward formal development of programs from algebraic specifications: implementations revisited. Acta Informatica 25, 233-281.

[ST 89] Sannella, D.T. and Tarlecki, A. Toward formal development of ML programs: foundations and methodology (long version). Report ECS-LFCS-89-71, Univ. of Edinburgh.

[SWi 83] Sannella, D. and Wirsing, M. A kernel language for algebraic specification and implementation. Proc. Intl. Conf. on Foundations of Computation Theory, Borgholm, Sweden, LNCS 158, 413-427.

[Sch 86] Schoett, O. Data abstraction and the correctness of modular programming. Ph.D. thesis, Univ. of Edinburgh.

[Wand 82] Wand, M. Specification, models, and implementations of data abstractions. Theoretical Computer Science $20,3-32$. 